УДК 517.5

E. A. Sevost'yanov, S. A. Skvortsov, I. A. Sverchevska

\title{
ON BOUNDARY EXTENSION OF ONE CLASS OF MAPPINGS IN TERMS OF PRIME ENDS
}

\begin{abstract}
E. A. Sevost'yanov, S. A. Skvortsov, I. A. Sverchevska. On boundary extension of one class of mappings in terms of prime ends, Mat. Stud. 53 (2020), 29-40.

Here we consider the classes of mappings of metric spaces that distort the modulus of families of paths similarly to Poletsky inequality. For domains, which are not locally connected at the boundaries, we obtain results on the boundary extension of the indicated mappings. We also investigate the local and global behavior of mappings in the context of the equicontinuity of their families. The main statements of the article are proved under the condition that the majorant responsible for the distortion of the modulus of the families of paths has a finite mean oscillation at the corresponding points. The results are applicable to well-known classes of conformal and quasiconformal mappings as well as mappings with a finite distortion.
\end{abstract}

1. Introduction. Recently we considered a number of problems related to the boundary extension of classes of mappings of metric spaces in the context of so-called prime ends, see [1]. In this article, we slightly strengthen the indicated results for the case when mappings satisfy somewhat more general conditions. Here, first of all, we mean the distortion of the module under the mapping, the order of which equals the Hausdorff dimension of the space in the previous publication. In addition, we refuse some separation conditions both on the maps themselves and on the spaces in which they act. In particular, in [1] we assumed that the mapped domain has a compact closure. Note that the last condition may be relaxed if it is required that the space of the action of the map admits the so-called weak sphericalization (see [2]). We note that the boundary extension of mappings in terms of prime ends has been studied recently by some other authors, see, e.g., [3], [4], [5] and [6].

Since all the necessary information related to the definition and properties of prime ends in metric spaces is given in [1], we allow ourselves not to provide them in this text. We also omit the part concerning Poincaré-type inequalities, Ahlfors regular spaces, and other information contained in the publication mentioned above. From now on we assume that the space $X$ is complete and supports a $p$-Poincaré inequality, and that the measure $\mu$ is doubling (see [4]). In this case, a space $X$ is locally connected (see [4, Section 2]), and proper (see [7, Proposition 3.1]). If $X$ is also connected then there exist constants $C>0$ and $q>0$ such that for all $x \in X, 0<r \leqslant R$ and $y \in B(x, R)$,

$$
\frac{\mu(B(y, r))}{\mu(B(x, R))} \leqslant C\left(\frac{r}{R}\right)^{q}
$$

2020 Mathematics Subject Classification: 30C65, 30L10, 31C12.

Keywords: metric spaces; quasiconformal mappings; mappings with bounded and finite distortion; equicontinuity; moduli of families of paths.

doi:10.30970/ms.53.1.29-40

(C) E. A. Sevost'yanov, S. A. Skvortsov, I. A. Sverchevska, 2020 
see $[4,(2.2)]$.

In what follows, $(X, d, \mu)$ and $\left(X^{\prime}, d^{\prime}, \mu^{\prime}\right)$ are metric spaces with metrics $d$ and $d^{\prime}$ and locally finite Borel measures $\mu$ and $\mu^{\prime}$, correspondingly. Sometimes instead of $(X, d, \mu)$ and $\left(X^{\prime}, d^{\prime}, \mu^{\prime}\right)$ we write $(X, d)$ and $\left(X^{\prime}, d^{\prime}\right)$, or even shorter, $X$ and $X^{\prime}$, if a misunderstanding is impossible. Given a metric space $(X, d, \mu)$ with a measure $\mu$, a domain in $X$ is an open path-connected set in $X$.

Recall, for a given continuous path $\gamma:[a, b] \rightarrow X$ in a metric space $(X, d)$, that its length is the supremum of the sums

$$
\sum_{i=1}^{k} d\left(\gamma\left(t_{i}\right), \gamma\left(t_{i-1}\right)\right)
$$

over all partitions $a=t_{0} \leqslant t_{1} \leqslant \ldots \leqslant t_{k}=b$ of the interval $[a, b]$. The path $\gamma$ is called rectifiable if its length is finite.

Given a family of paths $\Gamma$ in $X$, a Borel function $\rho: X \rightarrow[0, \infty]$ is called admissible for $\Gamma$, abbr. $\rho \in \operatorname{adm} \Gamma$, if

$$
\int_{\gamma} \rho d s \geqslant 1
$$

for all (locally rectifiable) $\gamma \in \Gamma$. Everywhere further, for any sets $E, F$, and $G$ in $X$, we denote by $\Gamma(E, F, G)$ the family of all paths $\gamma:[0,1] \rightarrow X$ such that $\gamma(0) \in E, \gamma(1) \in F$, and $\gamma(t) \in G$ for all $t \in(0,1)$. Everywhere further $(X, d, \mu)$ and $\left(X^{\prime}, d^{\prime}, \mu^{\prime}\right)$ are metric spaces with metrics $d$ and $d^{\prime}$ and locally finite Borel measures $\mu$ and $\mu^{\prime}$, correspondingly. We will assume that $\mu$ is a Borel measure such that $0<\mu(B)<\infty$ for all balls $B$ in $X$.

Given $p \geqslant 1$, the $p$-modulus of the family $\Gamma$ is the number

$$
M_{p}(\Gamma)=\inf _{\rho \in \operatorname{adm} \Gamma} \int_{X} \rho^{p}(x) d \mu(x) .
$$

Should adm $\Gamma$ be empty, we set $M_{p}(\Gamma)=\infty$. A family of paths $\Gamma_{1}$ in $X$ is said to be minorized by a family of paths $\Gamma_{2}$ in $X$, abbr. $\Gamma_{1}>\Gamma_{2}$, if, for every path $\gamma_{1} \in \Gamma_{1}$, there is a path $\gamma_{2} \in \Gamma_{1}$ such that $\gamma_{2}$ is a restriction of $\gamma_{1}$. In this case,

$$
\Gamma_{1}>\Gamma_{2} \quad \Rightarrow \quad M_{p}\left(\Gamma_{1}\right) \leq M_{p}\left(\Gamma_{2}\right)
$$

(see [8, Theorem 1]).

Put $\bar{X}:=X \cup\{\infty\}$, and let $h: \bar{X} \times \bar{X} \rightarrow \mathbb{R}$ be some metric. We say that $h$ satisfies the weak sphericalization condition, if $(\bar{X}, h)$ is a compact metric space, and $h$ and $d$ generate the same $X$ topology (note that this definition is slightly different from what we gave in [2]). Note that the extended space $\bar{X}$ with the metric $h$ introduced on it is a kind of analogue of the Riemannian sphere in the indicated sense.

If the space $X$ admits a weak sphericalization, then for a domain $G \subset \bar{X}$ and sets $E, F \subset G$, we put

$$
M_{p}(\Gamma(E, F, G)):=M_{p}(\Gamma(E \backslash\{\infty\}, F \backslash\{\infty\}, G \backslash\{\infty\})) .
$$

If $\Gamma$ is a family of paths in $\bar{X}$, we put $M_{p}(\Gamma)=M_{p}\left(\Gamma^{*}\right)$, where $\Gamma^{*}$ consists of those and only those paths of $\Gamma$, not passing through $\infty$. 
Let $G$ be a domain in $X$, let $Q: G \rightarrow[0, \infty]$ be a function measurable with respect to measure $\mu$ and $p, q \geqslant 1$ be fixed numbers. Put

$$
A\left(\zeta_{0}, r_{1}, r_{2}\right)=\left\{x \in X: r_{1}<d\left(x, \zeta_{0}\right)<r_{2}\right\}, \quad S_{i}=S\left(\zeta_{0}, r_{i}\right)=\left\{x \in X: d\left(x, \zeta_{0}\right)=r_{i}\right\} .
$$

Similarly to $\left[9\right.$, Ch. 7], a mapping $f: G \rightarrow X^{\prime}$ is called a ring Q-mapping at a point $x_{0} \in \bar{G}$ with respect to $(p, q)$-moduli, if for some $r_{0}=r_{0}\left(x_{0}\right)>0$ and for all $0<r_{1}<r_{2}<r_{0}$ the inequality

$$
M_{p}\left(f\left(\Gamma\left(S\left(\zeta_{0}, r_{1}\right), S\left(\zeta_{0}, r_{2}\right), G\right)\right)\right) \leqslant \int_{A \cap G} Q(x) \cdot \eta^{q}\left(d\left(x, \zeta_{0}\right)\right) d \mu(x)
$$

holds for any measurable function $\eta:\left(r_{1}, r_{2}\right) \rightarrow[0, \infty]$ with

$$
\int_{r_{1}}^{r_{2}} \eta(r) d r \geqslant 1
$$

Let $X$ and $Y$ be metric spaces. A mapping $f: X \rightarrow Y$ is discrete if $f^{-1}(y)$ is discrete for all $y \in Y$ and $f$ is open if it takes open sets onto open sets. Given a domain $D \subset X$, the cluster set of $f: D \rightarrow Y$ at $b \in \partial D$ is the set $C(f, b)$ of all points $z \in Y$ for which there exists a sequence $\left\{b_{k}\right\}_{k=1}^{\infty}$ in $D$ such that $b_{k} \rightarrow b$ and $f\left(b_{k}\right) \rightarrow z$ as $k \rightarrow \infty$. For a non-empty set $E \subset \partial D$ let $C(f, E)=\cup C(f, b)$, where $b$ ranges over set $E$. A mapping $f: G \rightarrow Y$ is closed in $G \subset X$ if $f(A)$ is closed in $f(G)$ whenever $A$ closed in $G$. A mapping $f$ is proper if $f^{-1}(K)$ is compact in $D$ whenever $K$ is a compact set of $f(D)$. A mapping $f$ is boundary preserving if $C(f, \partial D) \subset \partial f(D)$.

Let $D \subset X, f: D \rightarrow X^{\prime}$ be a discrete open mapping, $\beta:[a, b) \rightarrow X^{\prime}$ be a path, and $x \in f^{-1}(\beta(a))$. A path $\alpha:[a, c) \rightarrow D$ is called a maximal $f$-lifting of $\beta$ starting at $x$, if (1) $\alpha(a)=x$; (2) $f \circ \alpha=\left.\beta\right|_{[a, c)}$; (3) for $c<c^{\prime} \leqslant b$, there is no path $\alpha^{\prime}:\left[a, c^{\prime}\right) \rightarrow D$ such that $\alpha=\left.\alpha^{\prime}\right|_{[a, c)}$ and $f \circ \alpha^{\prime}=\left.\beta\right|_{\left[a, c^{\prime}\right)}$. If $X$ and $X^{\prime}$ are locally compact, $X$ is locally connected, and $f: D \rightarrow X^{\prime}$ is discrete and open, then there is a maximal $f$-lifting of $\beta$ starting at $x$, see $[2$, Lemma 2.1].

Let $G$ be a domain in a space $(X, d, \mu)$. Similarly to [10], we say that a function $\varphi: G \rightarrow \mathbb{R}$ has finite mean oscillation at a point $x_{0} \in \bar{G}$, abbr. $\varphi \in F M O\left(x_{0}\right)$, if

$$
\varlimsup_{\varepsilon \rightarrow 0} \frac{1}{\mu\left(B\left(x_{0}, \varepsilon\right)\right)} \int_{B\left(x_{0}, \varepsilon\right)}\left|\varphi(x)-\bar{\varphi}_{\varepsilon}\right| d \mu(x)<\infty
$$

where

$$
\bar{\varphi}_{\varepsilon}=\frac{1}{\mu\left(B\left(x_{0}, \varepsilon\right)\right)} \int_{B\left(x_{0}, \varepsilon\right)} \varphi(x) d \mu(x)
$$

is the mean value of the function $\varphi$ over the set $B\left(x_{0}, \varepsilon\right)=\left\{x \in G: d\left(x, x_{0}\right)<\varepsilon\right\}$ with respect to the measure $\mu$. Here the condition (5) includes the assumption that $\varphi$ is integrable with respect to the measure $\mu$ over the set $B\left(x_{0}, \varepsilon\right)$ for some $\varepsilon>0$.

In what follows, $\bar{D}_{P}$ is a completion of a domain $D$ by its prime ends, and $E_{D}$ : $=\bar{D}_{P} \backslash D$ denotes the set of all prime ends of $D$. We say that the boundary of the domain $G$ in $X$ ( or $\bar{X}$ ) is strongly accessible at a point $x_{0} \in \partial G$ with respect to p-modulus, if, for every 
neighborhood $U$ of the point $x_{0}$, there is a compact set $E \subset G$, a neighborhood $V \subset U$ of the point $x_{0}$ and a number $\delta>0$ such that

$$
M_{p}(\Gamma(E, F, G)) \geqslant \delta
$$

for every continuum $F \subset G$ intersecting $\partial U$ and $\partial V$. We say that the boundary $\partial G$ is strongly accessible with respect to p-modulus, if the corresponding property holds at every point of the boundary. The following result holds.

Theorem 1. Let $(X, d, \mu)$ and $\left(X^{\prime}, d^{\prime}, \mu^{\prime}\right)$ be metric spaces, and let $X^{\prime}$ admits a weak sphericalization. Let $D$ be a bounded domain in $X$ which is finitely connected at the boundary, let $D^{\prime}$ be a domain in $\overline{X^{\prime}}$, and let $1 \leqslant p<\infty, 1 \leqslant q<\infty$ be fixed numbers. Assume that $X$ is a complete space, supporting $q$-Poincaré inequality, and that the measure $\mu$ is doubling.

Let $Q: X \rightarrow(0, \infty)$ be a locally integrable function. Suppose that $f: D \rightarrow D^{\prime}, D^{\prime}=$ $f(D)$, is a discrete, closed and open ring $Q$-mapping with respect to $(p, q)$-moduli in $\partial D$. Moreover, suppose that $\partial D^{\prime}$ is strongly accessible with respect to $p$-modulus.

Then $f$ has a continuous extension $f: \bar{D}_{P} \rightarrow \overline{D^{\prime}}, f\left(\bar{D}_{P}\right)=\overline{D^{\prime}}$, whenever $Q \in F M O(\partial D)$.

In the above statement, the continuity of the map $f: \bar{D}_{P} \rightarrow \overline{D^{\prime}}$ is understood in the sense of an extended space $\left(\overline{X^{\prime}}, h\right)$, that is, it is stated that if the sequence $x_{k}, k=1,2, \ldots$, converges to the prime end $P_{0}$ as $k \rightarrow \infty$ in the metric space $\bar{D}_{P}$, then $f\left(x_{k}\right)$ converges to some value $y_{0} \in \overline{X^{\prime}}$ by the metric $h$. The statement, in this case, takes into account the metrizability of space $\bar{D}_{P}$ (see [4, Corollary 10.9]).

By correspondence $\left[E_{k}\right] \mapsto f\left(\left[E_{k}\right]\right),\left[E_{k}\right] \in E_{D}, f\left(\left[E_{k}\right]\right) \in \partial D^{\prime}$, we mean the following. If $\left\{x_{k}\right\}_{k=1}^{\infty}$ is a sequence with $x_{k} \rightarrow\left[E_{k}\right], k \rightarrow \infty$, then we set:

$$
f\left(\left[E_{k}\right]\right):=\lim _{k \rightarrow \infty} f\left(x_{k}\right) .
$$

The statement of the Theorem 1 includes that this limit exists, and it does not depend on a sequence $\left\{x_{k}\right\}_{k=1}^{\infty}$, which converges to $\left[E_{k}\right]$.

Remark 1. In fact, the FMO type condition that is present in Theorem 1 may be replaced by the following more general and more fundamental assumption, which will be used later in proving all the main results. Given a point $x_{0} \in \bar{D}$, assume that there exists a Lebesgue measurable function $\psi:(0, \infty) \rightarrow(0, \infty)$ such that

$$
I\left(\varepsilon, \varepsilon_{0}\right):=\int_{\varepsilon}^{\varepsilon_{0}} \psi(t) d t<\infty
$$

for every $\varepsilon \in\left(0, \varepsilon_{0}\right)$ and $I\left(\varepsilon, \varepsilon_{0}\right) \rightarrow \infty$ as $\varepsilon \rightarrow 0$, and

$$
\int_{\varepsilon<d\left(x, x_{0}\right)<\varepsilon_{0}} Q(x) \cdot \psi^{q}\left(d\left(x, x_{0}\right)\right) d \mu(x)=o\left(I^{q}\left(\varepsilon, \varepsilon_{0}\right)\right), \quad \varepsilon \rightarrow 0 .
$$

2. Main Lemma. The following statement was proved in [5, Lemma 5.1] for homeomorphisms in $\mathbb{R}^{2}$.

Lemma 1. Suppose that the assumptions of Theorem 1 are satisfied. Moreover, assume that, for every $x_{0} \in \partial D$ conditions (6)-(7) hold. Then $f$ has a continuous extension $f: \bar{D}_{P} \rightarrow{\overline{D^{\prime}}}^{\prime}$, $f\left(\bar{D}_{P}\right)=\overline{D^{\prime}}$. 
Proof. By [4, Corollary 10.9], $\bar{D}_{P}$ is metrizable. Now, by metrizability of $\bar{D}_{P}$, it is sufficient to prove that

$$
L=C(f, P):=\left\{y \in \overline{X^{\prime}}: y=\lim _{k \rightarrow \infty} f\left(x_{k}\right), x_{k} \rightarrow P, x_{k} \in D\right\}
$$

consists of single point $y_{0} \in \partial D^{\prime}$. Since $X^{\prime}$ is a space admitting a weak sphericalization, $L \neq \varnothing$. By [1, Proposition 2.1], $L \subset \partial D^{\prime}$.

Assume, to the contrary, that $f$ cannot be extended to $P$ continuously. Now, we can find at least two points $y_{0}$ and $z_{0} \in L$. Set $U=B\left(y_{0}, r_{0}\right)$, where $0<r_{0}<d\left(y_{0}, z_{0}\right)$. Now we may find sequences $y_{k}$ and $z_{k}$ in $f\left(E_{k}\right), k=1,2, \ldots, P=\left[E_{k}\right]$, such that $d\left(y_{0}, y_{k}\right)<r_{0}$ and $d\left(y_{0}, z_{k}\right)>r_{0}$ and, besides that, $y_{k} \rightarrow y_{0}$ and $z_{k} \rightarrow z_{0}$ as $k \rightarrow \infty$. By Remark 4.5 in [4] we may consider that the sets $E_{k}$ are open. Moreover, by Remark 2.6 in [4] the set $E_{k}$ is path connected for every $k \in \mathbb{N}$.

Denote $x_{0}:=I\left(\left[E_{k}\right]\right)$ (see $[4$, Theorem 10.8]). Now we show that, for every $r>0$ there exists $k \in \mathbb{N}$ such that

$$
E_{k} \subset B\left(x_{0}, r\right) \cap D
$$

Assume, to the contrary, that there exists $r>0$ with the following property: for every $k \in \mathbb{N}$ there exists $x_{k} \in E_{k} \backslash B\left(x_{0}, r\right)$. Since $\mu$ is doubling, $X$ is complete if and only if it is proper (i.e. every closed bounded set is compact), see [7, Proposition 3.1]. Since $D$ is bounded, $\bar{D}$ is compact. Now, we may find a subsequence $x_{k_{l}} \in D$ with $x_{k_{l}} \rightarrow \overline{x_{0}}$ as $l \rightarrow \infty$ for some $\overline{x_{0}} \in \bar{D}$. Given $i \in \mathbb{N}$, there exists $l_{0} \in \mathbb{N}$ such that $k_{l}>i$ for every $l \geqslant l_{0}$. Consequently, $x_{k_{l}} \in E_{k_{l}} \subset E_{i}$ for every $l \geqslant l_{0}$ and thus, $\overline{x_{0}} \in \overline{E_{i}}$. Since $i$ is arbitrary, we obtain that $\overline{x_{0}} \in \bigcap_{i=1}^{\infty} \overline{E_{i}}=\left\{x_{0}\right\}$. So, $x_{0}=\overline{x_{0}}$. It remains to show that $x_{k} \rightarrow x_{0}$ as $k \rightarrow \infty$. Assume the contrary, then there exists a subsequence $x_{m_{l}} \in D$ with $x_{m_{l}} \rightarrow \zeta_{0}$ as $l \rightarrow \infty$. Arguing as above, we obtain that $\zeta_{0}=x_{0}$, that disproves the contradiction mentioned above. Now $x_{k} \rightarrow x_{0}$ as $k \rightarrow \infty$ and thus, $x_{k} \in B\left(x_{0}, r\right)$. The inclusion (8) have been proved.

Since $y_{k}, z_{k} \in f\left(E_{k}\right)$, one can find at least two sequences $x_{k}, x_{k}^{\prime} \in E_{k}$ such that $f\left(x_{k}\right)=y_{k}$ and $f\left(x_{k}^{\prime}\right)=z_{k}$. By (8) $x_{k} \rightarrow x_{0}$ and $x_{k}^{\prime} \rightarrow x_{0}$ as $k \rightarrow \infty$. According to the definition of a strongly accessible boundary at a point $y_{0} \in \partial D^{\prime}$ with respect to $p$-modulus, for any neighborhood $U$ of this point one can find a compact set $C_{0}^{\prime} \subset \partial D^{\prime}$, a neighborhood $V$ of the point $y_{0}$ and a number $\delta>0$ such that

$$
M_{p}\left(\Gamma\left(C_{0}^{\prime}, F, D^{\prime}\right)\right) \geqslant \delta>0
$$

for an arbitrary continuum $F$ that intersects $\partial U$ and $\partial V$. By [1, Proposition 2.1] $C_{0}:=$ $f^{-1}\left(C_{0}^{\prime}\right)$ is compact subset of $D$. Consequently, $\delta_{0}=\operatorname{dist}\left(x_{0}, C_{0}\right)>0$. Then, without loss of generality, we may assume that $C_{0} \cap \overline{B\left(x_{0}, \varepsilon_{0}\right)}=\varnothing$. Since $E_{k}$ is connected, the points $x_{k}$ and $x_{k}^{\prime}$ can be connected by a path $\gamma_{k}$ lying in $E_{k}$.

As usually, given a path $\gamma:[a, b] \rightarrow X$, or $\gamma:[a, b] \rightarrow X^{\prime}$, we set

$$
|\gamma|:=\{x \in X: \exists t \in[a, b]: \gamma(t)=x\}
$$

Since $f\left(x_{k}\right)=y_{k} \in V$ and $f\left(x_{k}^{\prime}\right)=z_{k} \in D^{\prime} \backslash U$ for sufficiently large $k \in \mathbb{N}$, one can find a number $k_{0} \in \mathbb{N}$ such that, by virtue of (9),

$$
M_{p}\left(\Gamma\left(C_{0}^{\prime},\left|f\left(\gamma_{k}\right)\right|, D^{\prime}\right)\right) \geqslant \delta>0
$$


for all $k \geqslant k_{0}$. Let $\Gamma_{k}$ denote the family of all semi-open paths $\beta_{k}:[a, b) \rightarrow D^{\prime}$ such that $\beta(a) \in\left|f\left(\gamma_{k}\right)\right|, \beta_{k}(t) \in D^{\prime}$ for all $t \in[a, b)$, and

$$
\lim _{t \rightarrow b-0} \beta_{k}(t):=B_{k} \in C_{0}^{\prime}
$$

It is obvious that

$$
M_{p}\left(\Gamma_{k}\right)=M_{p}\left(\Gamma\left(C_{0}^{\prime},\left|f\left(\gamma_{k}\right)\right|, D^{\prime}\right)\right) .
$$

Since $X$ is a complete space, supporting $p$-Poincaré inequality, $X$ is proper and locally connected (see [4, Section 2], [7, Proposition 3.1]). Now, by [2, Lemma 2.1], each path $\beta_{k} \in \Gamma_{k}$ has a maximal lifting $\alpha_{*}$ in $D$ starting in $\left|\gamma_{k}\right|$. Let $\Gamma_{k}^{\prime}$ is a family of all maximal liftings $\alpha_{k}:[a, c) \rightarrow D$ of $\Gamma_{k}$ starting at $\left|\gamma_{k}\right|$. Note that no path $\alpha_{k} \in \Gamma_{k}^{\prime}, \alpha_{k}:[a, c) \rightarrow D$, can not tend to the boundary of $D$ as $t \rightarrow c-0$, since $C(f, \partial D) \subset \partial D^{\prime}$. Then $C\left(\alpha_{k}, c\right) \subset D$.

Now, assume that the $\alpha_{k}$ does not have a limit as $t \rightarrow c-0$. Consider

$$
G=\left\{x \in X: x=\lim _{k \rightarrow \infty} \alpha\left(t_{k}\right)\right\}, \quad t_{k} \in[a, c), \quad \lim _{k \rightarrow \infty} t_{k}=c .
$$

Letting to subsequences, if it is needed, we may restrict us by monotone sequences $t_{k}$. For $x \in G$, by continuity of $f, f\left(\alpha\left(t_{k}\right)\right) \rightarrow f(x)$ as $k \rightarrow \infty$, where $t_{k} \in[a, c), t_{k} \rightarrow c$ as $k \rightarrow \infty$. However, $f\left(\alpha\left(t_{k}\right)\right)=\beta\left(t_{k}\right) \rightarrow \beta(c)$ as $k \rightarrow \infty$. Thus, $f$ is a constant on $G$. On the other hand, $\bar{\alpha}$ is a compact set, because $\bar{\alpha}$ is a closed subset of the compact space $\bar{D}$ (see [11, Theorem 2.II.4, §41]). Now, by Cantor condition on the compact $\bar{\alpha}$, by monotonicity of $\alpha\left(\left[t_{k}, c\right)\right)$,

$$
G=\bigcap_{k=1}^{\infty} \overline{\alpha\left(\left[t_{k}, c\right)\right)} \neq \varnothing
$$

see $[11,1 . I I .4, \S 41]$. By [11, Theorem 5.II.5, §47], $G$ is connected. By discreteness of $f, G$ is a single-point set, $\alpha:[a, c) \rightarrow D$ extends to a closed path $\alpha:[a, c] \rightarrow D$, and $f(\alpha(c))=\beta(c)$.

Therefore, there exists

$$
\lim _{t \rightarrow c-0} \alpha_{k}(t)=A_{k} \in D .
$$

Observe that, in this case, by the definition of maximal lifting, we have $c=b$. Then, on the one hand,

$$
\lim _{t \rightarrow b-0} \alpha_{k}(t):=A_{k},
$$

and, on the other hand, by virtue of the continuity of the mapping $f$ in $D$,

$$
f\left(A_{k}\right)=\lim _{t \rightarrow b-0} f\left(\alpha_{k}(t)\right)=\lim _{t \rightarrow b-0} \beta_{k}(t)=B_{k} \in C_{0}^{\prime} .
$$

According to the definition of $C_{0}$, this implies that $A_{k}$ belongs to $C_{0}$. We imbed the compact set $C_{0}$ into a certain continuum $C_{1}$ lying completely in the domain $D$ (see Lemma 1 in [12]). Taking a smaller value of $\varepsilon_{0}>0$, we may again assume that $C_{1} \cap \overline{B\left(x_{0}, \varepsilon_{0}\right)}=\varnothing$. Now we have that $\Gamma_{k}^{\prime} \subset \Gamma\left(\left|\gamma_{k}\right|, C_{1}, D\right)$. Passing to a subsequence, if necessary, we may consider that $x_{k}$ and $x_{k}^{\prime} \in B\left(x_{0}, 2^{-k}\right)$. Observe that the function

$$
\eta(t)= \begin{cases}\psi(t) / I\left(2^{-k}, \varepsilon_{0}\right), & t \in\left(2^{-k}, \varepsilon_{0}\right), \\ 0, & t \in \mathbb{R} \backslash\left(2^{-k}, \varepsilon_{0}\right),\end{cases}
$$


where $I(\varepsilon):=\int_{\varepsilon}^{\varepsilon_{0}} \psi(t) d t$, satisfies a normalization condition of the form (6). By conditions (6) and $(7)$, we get

$$
M_{p}\left(f\left(\Gamma_{k}^{\prime}\right)\right) \leqslant M_{p}\left(f\left(\Gamma\left(\left|\gamma_{k}\right|, C_{1}, D\right)\right)\right) \leqslant \Delta(k),
$$

where $\Delta(k) \rightarrow 0$ as $k \rightarrow \infty$. However, $\Gamma_{k}=f\left(\Gamma_{k}^{\prime}\right)$. Therefore, using (12), we conclude that

$$
M_{p}\left(\Gamma_{k}\right)=M_{p}\left(f\left(\Gamma_{k}^{\prime}\right)\right) \leqslant \Delta(k) \rightarrow 0 \quad \text { as } \quad k \rightarrow \infty
$$

Relation (13), together with equality (11), contradicts inequality (10), which proves the possibility of continuous extension $f: \bar{D}_{P} \rightarrow \overline{D^{\prime}}$.

It remains to show that $f\left(\bar{D}_{P}\right)=\overline{D^{\prime}}$. It is clear, that $f\left(\bar{D}_{P}\right) \subset \overline{D^{\prime}}$. Now we show the inverse inclusion. Let $\zeta_{0} \in \overline{D^{\prime}}$. If $\zeta_{0} \in D^{\prime}$, then there exists $\xi_{0} \in D$ with $f\left(\xi_{0}\right)=\zeta_{0}$ and, consequently, $\zeta_{0} \in f(D)$. Assume that $\zeta_{0} \in \partial D^{\prime}$. Now there exists $\zeta_{m} \in D^{\prime}, \zeta_{m}=f\left(\xi_{m}\right)$, $\xi_{m} \in D$, such that $\zeta_{m} \rightarrow \zeta_{0}$ as $m \rightarrow \infty$. By [4, Theorem 10.10], $\bar{D}_{P}$ is a compact metric space. Now, we may consider that $\xi_{m} \rightarrow P_{0}$ as $m \rightarrow \infty$, where $P_{0}$ is some prime end in $\bar{D}_{P}$. Now $\zeta_{0} \in f\left(\bar{D}_{P}\right)$. The inclusion $\overline{D^{\prime}} \subset f\left(\bar{D}_{P}\right)$ has been proved. Consequently, $f\left(\bar{D}_{P}\right)=\overline{D^{\prime}}$.

3. Proof of the main result. We will say that a space $(X, d, \mu)$ is upper $\alpha$-regular at a point $x_{0} \in X$ if there is a constant $C>0$ such that

$$
\mu\left(B\left(x_{0}, r\right)\right) \leqslant C r^{\alpha}
$$

for the balls $B\left(x_{0}, r\right)$ centered at $x_{0} \in X$ with all radii $r<r_{0}$ for some $r_{0}>0$. We will also say that a space $(X, d, \mu)$ is upper $\alpha$-regular if the above condition holds at every point $x_{0} \in X$. The following statement holds (see [13, Lemma 2]).

Proposition 1. Let $G$ be a domain in Ahlfors upper q-regular metric space $(X, d, \mu), 1 \leqslant$ $q<\infty$. Assume that $x_{0} \in \bar{G}$ and $Q: G \rightarrow[0, \infty]$ belongs to $F M O\left(x_{0}\right)$. If

$$
\mu\left(G \cap B\left(x_{0}, 2 r\right)\right) \leqslant \gamma \cdot \log ^{q-2} \frac{1}{r} \cdot \mu\left(G \cap B\left(x_{0}, r\right)\right)
$$

for some $r_{0}>0$ and every $r \in\left(0, r_{0}\right)$, then $Q$ satisfies (7) at $x_{0}$ with $\psi(t):=\frac{1}{t \log \frac{1}{t}}$.

Proof of Theorem 1. follows from Lemma 1 and Proposition 1. Indeed, $X$ is upper regular by (1), and (14) holds because the measure $\mu$ is doubling by assumptions. So, the desired statement follows from the Lemma 1.

4. Equicontinuous families of homeomorphisms. Let us recall some definitions. Let $(X, d)$ and $\left(X^{\prime}, d^{\prime}\right)$ be metric spaces with distances $d$ and $d^{\prime}$, respectively. A family $\mathfrak{F}$ of mappings $f: X \rightarrow X^{\prime}$ is said to be equicontinuous at a point $x_{0} \in X$ if for every $\varepsilon>0$ there is $\delta>0$ such that

$$
d^{\prime}\left(f(x), f\left(x_{0}\right)\right)<\varepsilon
$$

for all $f \in \mathfrak{F}$ and $x \in X$ with $d\left(x, x_{0}\right)<\delta$. The family $\mathfrak{F}$ is equicontinuous if $\mathfrak{F}$ is equicontinuous at every point $x_{0} \in X$. In what follows, $X=\bar{D}_{P}$ and $d=m_{P}$, where $m_{P}$ is defined in [4, Corollary 10.9]. If we are talking about a family of mappings $f$ with values in the space $S$ that admits weak sphericalization $\bar{S}$, then in this case $X^{\prime}:=\bar{S}$ and $d^{\prime}=h$ is the corresponding metric in $\bar{S}$. The next definition can be found, e.g., in [14]. 
Suppose that the space $X^{\prime}$ admits a weak sphericalization and, moreover, $h$ is the corresponding metric in the extended space $\overline{X^{\prime}}$. Given $p \geqslant 1$, a domain $D \subset \overline{X^{\prime}}$ is called p-uniform domain if, for each $r>0$, there is $\delta>0$ such that

$$
M_{p}\left(\Gamma\left(F, F^{*}, D\right)\right) \geqslant \delta
$$

whenever $F$ and $F^{*}$ are continua of $D$ with $h(F) \geqslant r$ and $h\left(F^{*}\right) \geqslant r$. (Here $h(A):=$ $\left.\sup _{x, y \in A} h(x, y)\right)$. Domains $D_{i}, i \in I$, are said to be $p$-equi-uniform domains if, for $r>0$, the modulus condition above is satisfied by each $D_{i}$ with the same number $\delta$.

Assume that $X^{\prime}$ admits a weak sphericalization. Given $\delta>0, D \subset X$ and a measurable function $Q: D \rightarrow[0, \infty]$, denote $\mathfrak{R}_{Q, \delta, p, q}(D)$ the family of all ring $Q$-homeomorphisms $f: D \rightarrow \overline{X^{\prime}} \backslash K_{f}$ with respect to $(p, q)$-moduli in $D$, such that $f(D)$ is some open set in $\overline{X^{\prime}}$ and

$$
h\left(K_{f}\right)=\sup _{x, y \in K_{f}} h(x, y) \geqslant \delta,
$$

where $K_{f} \subset \overline{X^{\prime}}$ is a continuum. The following statement holds.

Lemma 2. Let $(X, d, \mu)$ and $\left(X^{\prime}, d^{\prime}, \mu^{\prime}\right)$ be metric spaces, let $D$ be a domain in $X$, and let $1 \leqslant p<\infty, 1 \leqslant q<\infty$ be fixed numbers.

Given $x_{0} \in D$, assume that conditions (6)-(7) hold. If $X$ is a locally path connected and locally compact space, $X^{\prime}$ admits a weak sphericalization and $\overline{X^{\prime}}$ is a uniform domain, then $\mathfrak{R}_{Q, \delta, p, q}(D)$ is equicontinuous at $x_{0}$. (As mentioned above, equicontinuity is understood here in the sense of extended metric space $\left.\left(\overline{X^{\prime}}, h\right)\right)$.

Proof. The idea of a proof is closely related to [15, Lemma 2]. Assume the contrary, i.e., assume that $\mathfrak{R}_{Q, \delta, p, q}(D)$ is not equicontinuous at $x_{0}$. Now, there is exists $x_{k} \in D$ and $f_{k} \in$ $\mathfrak{R}_{Q, \delta, p, q}(D)$ such that $x_{k} \rightarrow x_{0}$ as $k \rightarrow \infty$ and

$$
h\left(f_{k}\left(x_{k}\right), f_{k}\left(x_{0}\right)\right) \geqslant a_{0}, \quad k=1,2, \ldots,
$$

for some $a_{0}$. Since $X$ is locally connected by assumption, there is a sequence of balls $B\left(x_{0}, \varepsilon_{k}\right)$, $k=0,1,2, \ldots, \varepsilon_{k} \rightarrow 0$ as $k \rightarrow \infty$, such that

$$
V_{k+1} \subset \overline{B\left(x_{0}, \varepsilon_{k}\right)} \subset V_{k},
$$

where $V_{k}$ are connected neighborhoods of $x_{0}$ and $\overline{V_{k}}$ are continua in $D$. There is no loss of generality in assuming that $x_{k} \in V_{k}$. Now, $x_{0}$ and $x_{k}$ can be joined by a path $\gamma_{k}$ in the domain $V_{k}$. Note that an arbitrary path $\gamma \in \Gamma\left(K_{f_{k}},\left|f_{k}\left(\gamma_{k}\right)\right|, X^{\prime}\right)$ is not included entirely both in $f_{k}\left(B\left(x_{0}, \varepsilon_{0}\right)\right)$ and $X^{\prime} \backslash f_{k}\left(B\left(x_{0}, \varepsilon_{0}\right)\right)$, therefore there exists

$$
y_{1} \in|\gamma| \cap f_{k}\left(S\left(x_{0}, \varepsilon_{0}\right)\right)
$$

(see [11, Theorem 1, $\S 46$, item I]). Let $\gamma:[0,1] \rightarrow X^{\prime}$ and let $t_{1} \in(0,1)$ be such that $\gamma\left(t_{1}\right)=y_{1}$. There is no loss of generality in assuming that

$$
|\gamma|_{\left[0, t_{1}\right)} \mid \subset f_{k}\left(B\left(x_{0}, \varepsilon_{0}\right)\right) \text {. }
$$

We put $\gamma_{1}:=\left.\gamma\right|_{\left[0, t_{1}\right]}$, and $\alpha_{1}=f_{k}^{-1}\left(\gamma_{1}\right)$. Observe that $\left|\alpha_{1}\right| \subset B\left(x_{0}, \varepsilon_{0}\right)$, moreover, $\alpha_{1}$ is not included entirely either in $\overline{B\left(x_{0}, \varepsilon_{k-1}\right)}$, or in $X \backslash \overline{B\left(x_{0}, \varepsilon_{k-1}\right)}$. Consequently, there exists $t_{2} \in\left(0, t_{1}\right)$ with $\alpha_{1}\left(t_{2}\right) \in S\left(x_{0}, \varepsilon_{k-1}\right)$ (see [11, Theorem $1, \S 46$, item I]). There is no loss of generality in assuming that

$$
\left|\alpha_{1}\right|_{\left[t_{2}, t_{1}\right]} \mid \subset X \backslash \overline{B\left(x_{0}, \varepsilon_{k-1}\right)} .
$$

Put $\alpha_{2}=\left.\alpha_{1}\right|_{\left[t_{2}, t_{1}\right]}$. Observe that $\gamma_{2}:=f_{k}\left(\alpha_{2}\right)$ is a subcurve of $\gamma$. By the said above,

$$
\Gamma\left(K_{f_{k}},\left|f_{k}\left(\gamma_{k}\right)\right|, \overline{X^{\prime}}\right)>\Gamma\left(f_{k}\left(S\left(x_{0}, \varepsilon_{k-1}\right)\right), f_{k}\left(S\left(x_{0}, \varepsilon_{0}\right)\right), f_{k}(A)\right),
$$


where $A=A\left(x_{0}, \varepsilon_{k-1}, \varepsilon_{0}\right)$, and by (2) we obtain

$$
M_{p}\left(\Gamma\left(K_{f_{k}},\left|f_{k}\left(\gamma_{k}\right)\right|, \overline{X^{\prime}}\right)\right) \leqslant M_{p}\left(\Gamma\left(f_{k}\left(S\left(x_{0}, \varepsilon_{k-1}\right)\right), f_{k}\left(S\left(x_{0}, \varepsilon_{0}\right)\right), f_{k}(A)\right)\right) .
$$

Since $I\left(\varepsilon, \varepsilon_{0}\right) \rightarrow \infty$ as $\varepsilon \rightarrow 0$, we may consider that $I\left(\varepsilon_{k}, \varepsilon_{0}\right)>0$ for every $k=1,2, \ldots$ Consider the family of measurable functions

$$
\eta_{k}(t)=\psi(t) / I\left(\varepsilon_{k}, \varepsilon_{0}\right), \quad t \in\left(\varepsilon, \varepsilon_{0}\right) .
$$

Observe that $\int_{\varepsilon_{k}}^{\varepsilon_{0}} \eta_{k}(t) d t=1$. Now, by (3), (7) and (16), we obtain that

$$
M_{p}\left(\Gamma\left(K_{f_{k}},\left|f_{k}\left(\gamma_{k}\right)\right|, \overline{X^{\prime}}\right)\right) \leqslant \varphi\left(\varepsilon_{k}\right)
$$

where $\varphi$ is some function with $\varphi\left(\varepsilon_{k}\right) \rightarrow 0$ as $k \rightarrow \infty$. On the other hand, it follows from (15) that

$$
\min \left\{h\left(K_{f_{k}}\right), h\left(\left|f_{k}\left(\gamma_{k}\right)\right|\right)\right\} \geqslant r_{0}
$$

for some $r_{0}>0$ and every $k=1,2, \ldots$, . Now, since $X^{\prime}$ is uniformly domain, we obtain that

$$
M_{p}\left(\Gamma\left(K_{f_{k}},\left|f_{k}\left(\gamma_{k}\right)\right|, \overline{X^{\prime}}\right)\right) \geqslant \delta_{0}
$$

for some $\delta_{0}>0$ and every $k=1,2, \ldots$, . Now, (18) contradicts with (17). Thus, $\mathfrak{R}_{Q, \delta, p, q}(D)$ is equicontinuous at $x_{0}$, as required.

Assume that $X^{\prime}$ admits a weak sphericalization. Given $\delta>0, D \subset X$, a continuum $A \subset D$ and a measurable function $Q: D \rightarrow[0, \infty]$, denote $\mathfrak{F}_{Q, \delta, A, p, q}(D)$ the family of all ring $Q$-homeomorphisms $f: D \rightarrow \overline{X^{\prime}} \backslash K_{f}$ with respect to $(p, q)$-modulus in $D$, such that $f(D)$ is some open set in $\overline{X^{\prime}}$,

$$
h\left(K_{f}\right)=\sup _{x, y \in K_{f}} h(x, y) \geqslant \delta \text { and } h(f(A)) \geqslant \delta,
$$

where $K_{f} \subset \overline{X^{\prime}}$ is some continuum. An analog of the following result was proved in [14, Theorem 3.1] (see also [1, Lemma 5.2]).

Lemma 3. Let $(X, d, \mu)$ and $\left(X^{\prime}, d^{\prime}, \mu^{\prime}\right)$ be metric spaces, and let $X^{\prime}$ admits a weak sphericalization. Let $D$ be a bounded domain in $X$ which is finitely connected at the boundary, let $D^{\prime}$ be a domain in $\overline{X^{\prime}}$, and let $1 \leqslant p<\infty, 1 \leqslant q<\infty$ be fixed numbers. Assume that $X$ is a complete space, supporting $p$-Poincaré inequality, and that the measure $\mu$ is doubling.

Let $Q: X \rightarrow(0, \infty)$ be a locally integrable function. Assume that, for every $x_{0} \in \bar{D}$ conditions (6)-(7) hold. If $D_{f}^{\prime}:=f(D)$ and $\overline{X^{\prime}}$ are equi-uniform domains over $f \in \mathfrak{F}_{Q, \delta, A, p, q}(D)$, then every $f \in \mathfrak{F}_{Q, \delta, A, p, q}(D)$ has a continuous extension $f: \bar{D}_{P} \rightarrow \overline{D_{f}^{\prime}}$, and $\mathfrak{F}_{Q, \delta, A, p, q}(D)$ is equicontinuous in $\bar{D}_{P}$.

Proof. Observe that $\partial D_{f}^{\prime}=\partial f(D)$ is strongly accessible for every $f \in \mathfrak{F}_{Q, \delta, A, p, q}(D)$. Indeed, assume that $x_{0} \in \partial D_{f}^{\prime}$. Given a neighborhood $U$ of $x_{0}$, there exists $\varepsilon_{1}>0$ such that

$$
V=B\left(x_{0}, \varepsilon_{1}\right), V:=\overline{B\left(x_{0}, \varepsilon_{1}\right)} \subset U .
$$

Assume that $\partial U \neq \varnothing$. Now, $\partial V \neq \varnothing$ by connectedness of $\overline{X^{\prime}}$ (see $[11,5.1 .46]$ ). Now, we set $\varepsilon_{2}:=h^{\prime}(\partial U, \partial V)>0$. Since $D_{f}^{\prime}$ are equi-uniform, we obtain that

$$
M_{p}\left(\Gamma\left(F, G, D_{f}^{\prime}\right)\right) \geqslant \delta>0
$$


for some $\delta>0$ whenever $F$ and $G$ are continua in $D_{f}^{\prime}$ with

$$
F \cap \partial U \neq \varnothing \neq F \cap \partial V \text { and } G \cap \partial U \neq \varnothing \neq G \cap \partial V
$$

(because, in this case, we obtain that $h(F) \geqslant \varepsilon_{2}$ and $h(G) \geqslant \varepsilon_{2}$ ). Thus, $\partial D_{f}^{\prime}=f(D)$ is strongly accessible with respect to $p$-modulus, as required. Now, by Lemma $1, f \in$ $\mathfrak{F}_{Q, \delta, A, p, q}(D)$ has a continuous extension $f: \bar{D}_{P} \rightarrow \overline{D_{f}^{\prime}}$.

Since $\mu$ is doubling, $X$ is complete if and only if it is proper (i.e. every closed bounded set is compact), see [7, Proposition 3.1]. Now, $X$ is a locally compact space. Since $X$ is complete, $X$ supports an $q$-Poincaré inequality, and the measure $\mu$ is doubling, we obtain that $X$ is locally connected (see [4], see also [16, Theorem 17.1]). Moreover, $X$ is locally path connected by the Mazurkiewicz-Moore-Menger theorem (see in [11, Theorem 1.6.50.II]). Thus, all conditions of Lemma 2 are satisfied. Now, by Lemma $2, \mathfrak{F}_{Q, \delta, A, p, q}(D)$ is equicontinuous at $x_{0}$ for every $x_{0} \in D$.

It remains to show that $\mathfrak{F}_{Q, \delta, A, p, q}(D)$ is equicontinuous on $E_{D}=\bar{D}_{P} \backslash D$. Assume the contrary, i.e., assume that there exists $P_{0} \in E_{D}$ such that $\mathfrak{F}_{Q, \delta, A, p, q}(D)$ is not equicontinuous at $P_{0}$. Now, there is exists $P_{k} \in \bar{D}_{P}$ and $f_{k} \in \mathfrak{F}_{Q, \delta, A}(D)$ such that $P_{k} \rightarrow P_{0}$ as $k \rightarrow \infty$ and

$$
h\left(f_{k}\left(P_{k}\right), f_{k}\left(P_{0}\right)\right) \geqslant a_{0}
$$

for some $a_{0}>0$. Since $f_{k}$ has a continuous extension on $\bar{D}_{P}$, given $k \in \mathbb{N}$, we can find $x_{k} \in D$ with $m_{P}\left(x_{k}, P_{k}\right)<1 / k$ and $h\left(f_{k}\left(x_{k}\right), f_{k}\left(P_{k}\right)\right)<1 / k$. Thus, we obtain from (19) that

$$
h\left(f_{k}\left(x_{k}\right), f_{k}\left(P_{0}\right)\right) \geqslant a_{0} / 2 \quad \forall \quad k=1,2, \ldots, .
$$

Similarly, we can find $x_{k}^{\prime} \in D$ such that $x_{k}^{\prime} \rightarrow P_{0}$ as $k \rightarrow \infty$, and

$$
h\left(f_{k}\left(x_{k}^{\prime}\right), f_{k}\left(P_{0}\right)\right)<1 / k, k=1,2, \ldots
$$

Thus, we obtain from (20) that

$$
h\left(f_{k}\left(x_{k}\right), f_{k}\left(x_{k}^{\prime}\right)\right) \geqslant a_{0} / 4 \quad \forall \quad k=1,2, \ldots,
$$

where $x_{k}$ and $x_{k}^{\prime} \in D$ satisfy conditions $x_{k} \rightarrow P_{0}, x_{k}^{\prime} \rightarrow P_{0}$ as $k \rightarrow \infty$.

Let $P_{0}=\left[E_{k}\right]$. Denote $x_{0}:=I\left(\left[E_{k}\right]\right)$ (see [4, Theorem 10.8]). By [4, Remark 4.5] we may consider that the sets $E_{k}$ are open. Moreover, by Remark 2.6 in [4] the set $E_{k}$ is path connected for every $k \in \mathbb{N}$. Arguing as in the proof of Lemma 1, we may show that, for every $r>0$ there exists $k \in \mathbb{N}$ such that

$$
E_{k} \subset B\left(x_{0}, r\right) \cap D .
$$

Thus, there is no loss of generality in assuming that

$$
x_{k}, x_{k}^{\prime} \in E_{k} \text { and } E_{k} \subset B\left(x_{0}, 2^{-k}\right) .
$$

Let $\gamma_{k}$ be a path, joining $x_{k}$ and $x_{k}^{\prime}$ in $E_{k}$. Observe that

$$
A \subset D \backslash B\left(x_{0}, 2^{-k}\right)
$$

for all $k>k_{0}$ and some $k_{0} \in \mathbb{N}$. We may consider that $2^{-k_{0}}<\varepsilon_{0}$. Let $\Gamma_{k}$ be a family of paths joining $\left|\gamma_{k}\right|$ and $A$ in $D$. By (2), we obtain that

$$
M_{p}\left(f_{k}\left(\Gamma_{k}\right)\right) \leqslant M_{p}\left(f_{k}\left(\Gamma\left(S\left(x_{0}, 2^{-k}\right), S\left(x_{0}, 2^{-k_{0}}\right), A\left(x_{0}, 2^{-k}, 2^{-k_{0}}\right)\right)\right)\right) .
$$

Observe that

$$
\eta(t)=\left\{\begin{array}{ll}
\psi(t) / I\left(2^{-k}, 2^{-k_{0}}\right), & t \in\left(2^{-k}, 2^{-k_{0}}\right), \\
0, & t \in \mathbb{R} \backslash\left(2^{-k}, 2^{-k_{0}}\right),
\end{array} \quad I\left(\varepsilon, \varepsilon_{0}\right):=\int_{\varepsilon}^{\varepsilon_{0}} \psi(t) d t\right.
$$


satisfies the condition (4) for $r_{1}=2^{-k}$ and $r_{2}=2^{-k_{0}}$. By the definition of a ring $Q$ homeomorphism at a boundary point $x_{0},(7)$ and (22) imply

$$
M_{p}\left(f_{k}\left(\Gamma_{k}\right)\right) \leqslant \alpha\left(2^{-k}\right) \rightarrow 0
$$

as $k \rightarrow \infty$, where $\alpha(\varepsilon)$ is some nonnegative function with $\alpha(\varepsilon) \rightarrow 0$ as $\varepsilon \rightarrow 0$.

However,

$$
f_{k}\left(\Gamma_{k}\right)=\Gamma\left(\left|f_{k}\left(\gamma_{k}\right)\right|, f_{k}(A), D_{f_{k}}^{\prime}\right) .
$$

By assumption, $h\left(f_{k}(A)\right) \geqslant \delta, k=1,2, \ldots$, moreover, by (21) we obtain that

$$
h\left(\left|f_{k}\left(\gamma_{k}\right)\right|\right) \geqslant a_{0} / 4, k=1,2, \ldots
$$

Since $D_{f_{k}}^{\prime}$ are are equi-uniform domains, we obtain that

$$
M_{p}\left(f_{k}\left(\Gamma_{k}\right)\right) \geqslant r_{0}, k=1,2, \ldots,
$$

for some $r_{0}>0$. But $(24)$ contradicts $(23)$. Thus, $\mathfrak{F}_{Q, \delta, A, p, q}(D)$ is equicontinuous at $P_{0}$, as required.

The following main result holds.

Theorem 2. Let $(X, d, \mu)$ and $\left(X^{\prime}, d^{\prime}, \mu^{\prime}\right)$ be metric spaces, and let $X^{\prime}$ admits a weak sphericalization. Let $D$ be a bounded domain in $X$ which is finitely connected at the boundary, let $D^{\prime}$ be a domain in $\overline{X^{\prime}}$, and let $1 \leqslant p<\infty, 1 \leqslant q<\infty$ be fixed numbers. Assume that $X$ is a complete space, supporting $p$-Poincaré inequality, and that the measure $\mu$ is doubling.

Let $Q: X \rightarrow(0, \infty)$ be a locally integrable function. Assume that $Q \in F M O(\bar{D})$. If $D_{f}^{\prime}:=$ $f(D)$ and $\overline{X^{\prime}}$ are equi-uniform domains over $f \in \mathfrak{F}_{Q, \delta, A, p, q}(D)$, then every $f \in \mathfrak{F}_{Q, \delta, A, p, q}(D)$ has a continuous extension $f: \bar{D}_{P} \rightarrow \overline{D_{f}^{\prime}}$, and $\mathfrak{F}_{Q, \delta, A, p, q}(D)$ is equicontinuous in $\bar{D}_{P}$.

Proof of Theorem 2. follows from Lemma 3 and Proposition 1. Indeed, $X$ is upper regular by (1), and (14) holds because the measure $\mu$ is doubling by assumptions. So, the desired statement follows from Lemma 3.

\section{REFERENCES}

1. E. Sevost'yanov, On boundary extension of mappings in metric spaces in terms of prime ends, Ann. Acad. Sci. Fenn. Math., 44 (2019), №1, 65-90.

2. E. Sevost'yanov, A. Markysh, On Sokhotski-Casorati-Weierstrass theorem on metric spaces, Complex Variables and Elliptic Equations, 64 (2019), №12, 1973-1993.

3. T. Adamowicz, Prime ends in metric spaces and quasiconformal-type mappings, Analysis and Mathematical Physics, https://doi.org/10.1007/s13324-019-00292-z.

4. T. Adamowicz, A. Björn, J. Björn, N. Shanmugalingam, Prime ends for domains in metric spaces, Adv. Math., 238 (2013), 459-505.

5. V.Ya. Gutlyanskii, V.I. Ryazanov, E. Yakubov, The Beltrami equations and prime ends, Ukr. Mat. Visn. 12 (2015), №1, 27-66; transl. J. Math. Sci. (N.Y.) 210 (2015), №1, 22-51.

6. D.A. Kovtonyuk, V.I. Ryazanov, On the theory of prime ends for space mappings, Ukr. Mat. Zh., 67 (2015), №4, 467-479; Engl. transl. Ukrainian Math. J., 67 (2015), №4, 528-541.

7. A. Björn, J. Björn, Nonlinear Potential Theory on Metric Spaces, in EMS Tracts in Mathematics, V.17, European Math. Soc., Zurich, 2011.

8. B. Fuglede, Extremal length and functional completion, Acta Math., 98 (1957), 171-219. 
9. O. Martio, V. Ryazanov, U. Srebro, E. Yakubov, Moduli in Modern Mapping Theory, Springer Monographs in Mathematics, Springer, New York etc., 2009.

10. A. Ignat'ev, V. Ryazanov, Finite mean oscillation in mapping theory (Russian), Ukr. Mat. Visn., 2 (2005), №3, 395-417, Engl. translation Ukr. Math. Bull., 2 (2005), №3, 403-424.

11. K. Kuratowski, Topology, v. 2, Academic Press, New York-London, 1968.

12. E.S. Smolovaya, Boundary behavior of ring Q-homeomorphisms in metric spaces, Ukrainian Math. J., 62 (2010), №5, 785-793.

13. E.S. Afanas'eva, On boundary behavior of one class of mappings in metric spaces, Ukrainian Math. Journ., 66 (2014), №1, 16-29.

14. R. Näkki, B. Palka, Uniform equicontinuity of quasiconformal mappings, Proc. Amer. Math. Soc., 37 (1973), №2, 427-433.

15. E. Sevost'yanov, Local and boundary behavior of maps in metric spaces, Algebra i analiz, 28 (2016), №6, 118-146 (in Russian); Engl. translation St. Petersburg Math. J., 28 (2017), №6, 807-824.

16. J. Cheeger, Differentiability of Lipschitz functions on metric measure spaces, Geom. Funct. Anal., 9 (1999), 428-517.

Zhytomyr Ivan Franko State University

Zhytomyr, Ukraine

Institute of Applied Mathematics and Mechanics

of NAS of Ukraine,

Slavyansk, Ukraine

esevostyanov2009@gmail.com

Zhytomyr Ivan Franko State University

Zhytomyr, Ukraine

serezha.skv@gmail.com

sverchevska.ia@gmail.com 